\title{
Compliance with CPAP therapy in patients with the sleep apnoea/hypopnoea syndrome
}

\author{
Heather M Engleman, Sascha E Martin, Neil J Douglas
}

\begin{abstract}
Background - Continuous positive airway pressure (CPAP) therapy is the treatment of choice for the sleep apnoea/ hypopnoea syndrome. Compliance with this relatively obtrusive therapy has not been well studied.

Methods - Usage of CPAP was investigated in 54 patients with sleep apnoea/ hypopnoea syndrome (median 36 (range 7-129) apnoeas + hypopnoeas/hour slept) over the first 1-3 months after starting CPAP therapy. In all cases CPAP usage was monitored by hidden time clocks that indicated for how long the machines were switched on - that is, the CPAP run time. In 32 patients the time at which the CPAP mask pressure was at the therapeutic level of CPAP pressure set for that patient - that is, the mask time - was also monitored. In all patients objective daytime sleepiness was assessed by multiple sleep latency before and after CPAP therapy.
\end{abstract}

Results - The mean (SE) nightly CPAP run time was $4 \cdot 7(0.4)$ hours. There was no correlation between run time and severity of the sleep apnoea/hypopnoea syndrome as assessed by apnoea + hypopnoea frequency or multiple sleep latency, and no correlation between CPAP usage and improvement in multiple sleep latency. Thirty two patients in whom mask time was recorded had therapeutic CPAP pressures for $89 \%(3 \%)$ of their CPAP run times. Patients who experienced side effects from CPAP used their CPAP machines significantly less than those who did not.

Conclusions - Patients with sleep apnoea/ hypopnoea syndrome used CPAP for less than five hours/night on average with no correlation between severity of sleep apnoea/hypopnoea syndrome and CPAP usage. Patients who complained of side effects used their CPAP therapy less. It is recommended that, as a minimum, CPAP run time should be regularly recorded in all patients receiving CPAP therapy.

(Thorax 1994;49:263-266)

Recent evidence indicates that the sleep apnoea/hypopnoea syndrome has a prevalence in middle age of $2 \%$ in women and $4 \%$ in men, ${ }^{1}$ approaching the approximately $6 \%$ prevalence of asthma in the middle aged population. ${ }^{23}$ The treatment of choice for the sleep apnoea/hypopnoea syndrome is continuous positive airway pressure (CPAP) therapy via the nose.

Compliance with therapy in patients with airflow obstruction is relatively poor. ${ }^{4-6} \mathrm{De}$ spite the fact that CPAP therapy is more obtrusive than the use of bronchodilator inhalers, and that CPAP therapy carries a relatively high initial capital cost, there have been few studies of compliance with CPAP therapy in patients with the sleep apnoea/ hypopnoea syndrome and no studies in a British population. We have therefore studied CPAP use in such patients, and have also examined whether there were correlations between indices of severity of the sleep apnoea/ hypopnoea syndrome and objective measures of compliance.

\section{Methods}

Usage of CPAP was studied in 54 patients during the first 1-3 months after initiation of CPAP therapy.

\section{PATIENTS}

Of the 54 patients studied, 48 were men. The mean (SE) age was $51(1.3)$ years and body mass index was $33(1 \cdot 1) \mathrm{kg} / \mathrm{m}^{2}$. Each had at least two major symptoms of the sleep apnoea/ hypopnoea syndrome ${ }^{7}$ plus at least five apnoeas + hypopnoeas/hour of sleep ${ }^{8}$ recorded by overnight polysomnographic monitoring performed with our usual equipment and scoring techniques. ${ }^{9}$ These patients were deliberately selected to provide a wide range of frequencies of apnoeas + hypopnoeas (median 36 (range 7-129)/hour slept).

All patients had multiple sleep latency tests performed ${ }^{1011}$ in the baseline state and again, off CPAP, after the last night of CPAP therapy. Sleep onset was defined by the first 20 second epoch of any sleep stage. ${ }^{12}$

\section{CPAP THERAPY}

Following an initial positive clinical sleep study, all patients had the rationale for CPAP therapy explained to them and the CPAP equipment demonstrated. They then had an overnight CPAP titration sleep study in which the CPAP pressure was adjusted to the minimum pressure that normalised breathing pattern and minimised electroencephalographic arousals, defined as an episode of alpha or theta rhythm for at least 1.5 seconds associated with a transient increase in electromyographic tone, however brief. ${ }^{13}$ In the morning the patients' further queries about CPAP therapy were 
answered and they were allowed home with an appropriately set CPAP unit and a correctly fitting mask or intranasal system. The importance of all night use of the CPAP machines was stressed. The patients were given a telephone number to contact if they experienced any problems with their CPAP machines or side effects, and were phoned after two weeks on CPAP to check their progress.

\section{PROTOCOLS}

The first 22 patients studied received Respironics Sleep Easy II or Sleep Easy III CPAP units with hidden time clocks which indicated for how long the machines were switched on the "CPAP run time." These time clocks were read when the machines were issued and reread after three months. These patients were taking part in a parallel group study of the effect of CPAP on daytime function. ${ }^{11}$

The 32 subsequent patients were issued with ResCare Sullivan APD 1 CPAP units which had been modified by building in two time clocks. The first, as in the Sleep Easy II and III machines, indicated the CPAP run time. The second was linked to a pressure sensitive switch connected via a polythene tube to the CPAP mask, this tube passing along the CPAP hosing. ${ }^{14}$ The pressure sensitive switch was set so that it was activated when the pressure in the mask was more than $2 \mathrm{~cm} \mathrm{H}_{2} \mathrm{O}$ below the therapeutic level of CPAP pressure set for that patient, thus monitoring the time spent at an effective CPAP pressure "the mask time." These patients were taking part in a crossover trial of CPAP therapy; their time clocks were read when the CPAP units were dispensed and again one month thereafter.

\section{STATISTICAL ANALYSIS}

Correlation analysis to determine significant associations and multiple regression analysis with forward stepwise entry of variables to assess the relative contributions of different factors were conducted using the SPSS-X package. ${ }^{15}$

\section{Results}

The mean (SE) nightly CPAP run time for the 54 patients was $4.7(0.4)$ hours. There was no correlation between CPAP run time and frequency of apnoeas + hypopnoeas (fig 1), baseline multiple sleep latency time (MSLT; fig 2), or body mass index, nor was there any correlation between change in MSLT following therapy and CPAP run time (fig 3). Separate analyses of the data from the two component studies showed that none of these correlations were significant.

The 32 patients in whom mask time was recorded had therapeutic CPAP pressures for $89 \%(3 \%)$ of their CPAP run times (fig 4$)$. The patient in whom appropriate CPAP pressure was only achieved for $24 \%$ of the night was subsequently found to have displaced the cap from one of the sensing ports on his CPAP mask.

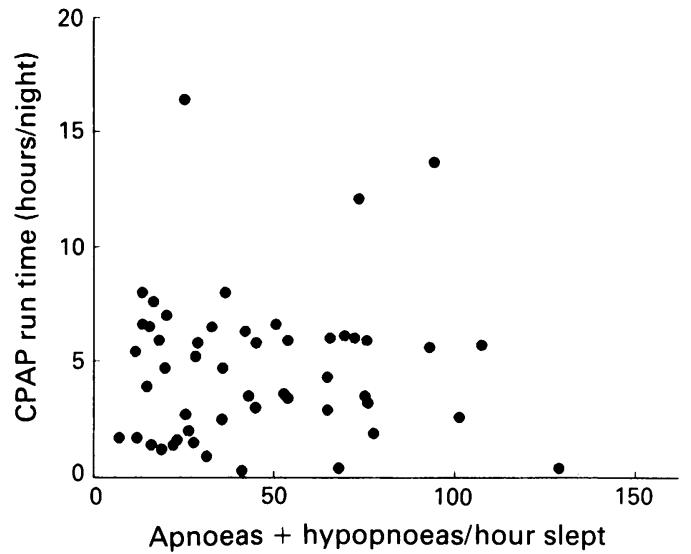

Figure 1 Relation between number of apnoeas + hypopnoeas/hour slept and CPAP run time. No significant correlation was seen $(r=0.04, p<0.5)$.

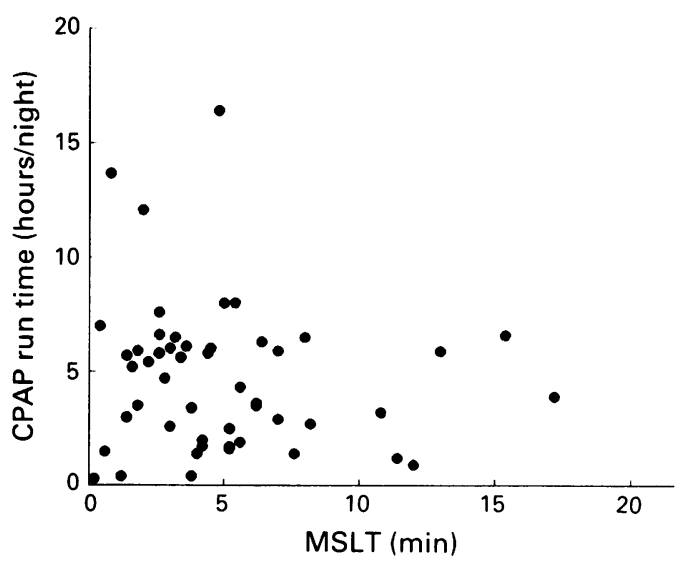

Figure 2 Relation between mean multiple sleep latency time (MSLT) at baseline and CPAP run time. No significant correlation was seen $(r=0 \cdot 13, p<0 \cdot 2)$.

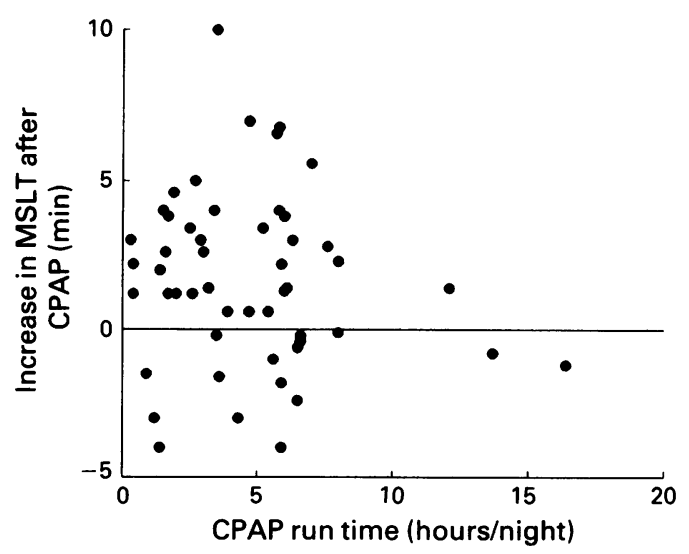

Figure 3 Relation between CPAP run time and improvement in multiple sleep latency time. No significant correlation was seen $(r=0 \cdot 13, p>0 \cdot 1)$. 


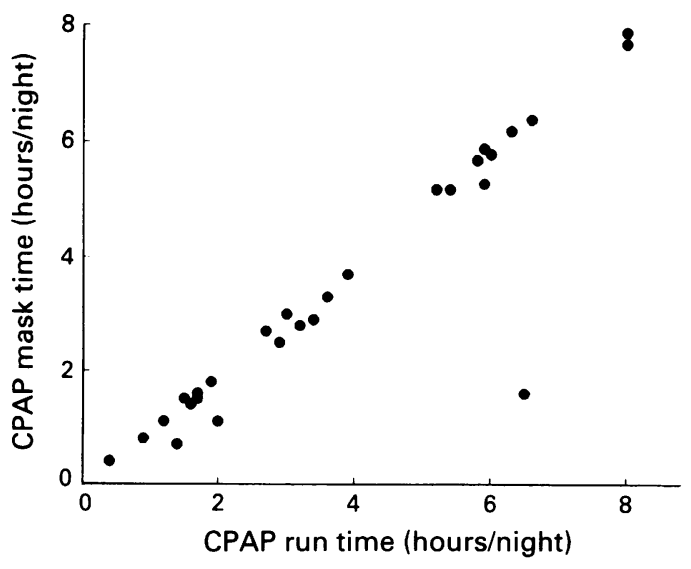

Figure 4 Relation between CPAP run time and CPAP mask time, the time at therapeutic CPAP pressure.

A total of 13 of these 32 patients (41\%) contacted our staff to report problems associated with the use of their CPAP units. Nine complained of nasal congestion and were prescribed steroid nasal spray to relieve this side effect, two experienced dryness of the upper airway which was treated by humidification of the CPAP airstream, and five patients disliked the pressure effects of the CPAP system. Average CPAP mask time was higher in the patients reporting no problems with CPAP than in those reporting problems $(4 \cdot 1(0.6) v 2.4(0.4)$ hours/night; $\mathrm{p}=0.02)$, with a trend $(\mathrm{p}<0.1)$ towards higher CPAP run times in those patients reporting no problems $(4.3(0.6) v 2 \cdot 8$ $(0 \cdot 6)$ hours/night).

The therapeutic CPAP pressure assessed for each patient during the CPAP titration run and given to these patients during the studies correlated significantly with the frequency of apnoeas + hypopnoeas $(p<0.001)$, collar size $(\mathrm{p}<0.001)$, the male sex $(\mathrm{p}<0.02)$, and body mass index $(p<0.05)$. Multiple regression showed that the most important variables in determining CPAP pressure were collar size $(\mathrm{p}<0.005)$ and apnoea + hypopnoea frequency $(p<0.02)$, which together explain $53 \%$ of the variance in prescribed CPAP pressure.

\section{Discussion}

The average duration of CPAP use ( $4 \cdot 7$ hours/ night) seems disappointing. However, these results have to be compared with those obtained by similar objective methods in other chronic conditions. For example, asthmatic patients have been shown by electronic monitoring devices to use their chronic treatment as instructed on only $37 \%$ of days, ${ }^{6}$ and antiepileptic treatment was taken as prescribed on $39 \%$ of days in another study. ${ }^{16}$

The results in our study are very similar to those in a contemporaneous study performed in two centres in North America for which the average duration of CPAP used by 35 patients with sleep apnoea/hypopnoea syndrome was $4.9(2.0)$ hours/night, and the CPAP was received at the appropriate pressure for $91 \%$ of the CPAP run time. ${ }^{17}$ The subjects in that study only used their CPAP units for $66 \%$ of the nights, however, and we therefore deduce that their average CPAP use was $3 \cdot 2$ hours/ night. Similar results have been reported by another North American group. ${ }^{14}$ CPAP run times averaging $5 \cdot 1$ hours/night were obtained in a French sample of 45 patients. ${ }^{18}$ Our results extend these observations by examining correlates between CPAP usage and severity of the sleep apnoea/hypopnoea syndrome, and indicate that there was no correlation between any measure of severity of the sleep apnoea/hypopnoea syndrome and objective CPAP use. This somewhat surprising result means, not only that patients with severe sleep apnoea/hypopnoea syndrome cannot be relied upon to comply with their therapy, but also that some patients with mild sleep apnoea/hypopnoea syndrome in terms of apnoea + hypopnoea frequency or objective daytime sleepiness (MSLT) use their CPAP therapy regularly. Our results also extend these previous observations by reporting results in British patients in whom CPAP units were provided free of charge. Theoretically this might reduce motivation to use CPAP in comparison with patients who had to pay for their own units, but our CPAP usage is similar to that in North America. ${ }^{17}$

CPAP therapy is obtrusive, and it is not surprising that patients do not use it all night every night. However, the results in this study indicate that CPAP usage is extremely variable between patients and that, on average, CPAP use is less than five hours/night. Even with this level of compliance, however, these patients have reported improvements in symptoms and we have identified statistically significant improvements in objective daytime sleepiness which, in the first sample of 22 patients, rose to $5.5(0.4)$ minutes from a preCPAP level of 3.5 $(0 \cdot 7)$ minutes. ${ }^{11}$ It thus seems likely that the patients titrate their own CPAP use to provide an acceptable balance between the inconvenience of CPAP and the benefits of therapy.

The patients in whom mask time was monitored achieved satisfactory mask pressures for an average of $89 \%$ of the run time. These APD 1 CPAP units had built-in delay timers which allowed the patients to ramp up their CPAP pressure, achieving therapeutic CPAP pressure within 20 minutes of the machine being switched on. One would therefore anticipate that optimal CPAP pressure would only be achieved for $93 \%$ of the night in patients using a 20 minute delay on one occasion if the machine was switched on for the average 4.7 hours/night. It thus seems that these patients are achieving satisfactory CPAP pressures for most of the time during which the CPAP machines are switched on, with the one noticeable exception of the patient whose pressure monitor port cap was displaced. Thus, while monitoring the time at the appropriate CPAP pressure is the ideal method of monitoring compliance and manufacturers should work towards this end, recording the time for which the machine is switched on appears to give a good measure of CPAP use. We would recommend that, as a minimum, the machine run time should be regularly recorded in all patients receiving CPAP therapy.

Our study documents a relatively high inci- 
dence $(41 \%)$ of problems relating to CPAP use such as side effects and pressure intolerance. Such problems may have a serious impact on CPAP compliance rates, with mask times in patients reporting problems reduced to $60 \%$ of those found in patients not complaining of problems with CPAP. These findings emphasise the need for adequate follow up care and problem management for patients prescribed CPAP.

The patients reported formed part of two separate studies. ${ }^{11}$ We have reported their compliance data in this paper in order to allow aggregate data to be used to assess correlations (or lack of them) between CPAP use and severity of the sleep apnoea/hypopnoea syndrome. We do not believe that the difference in the study protocols will have influenced the results. In particular, our own evidence is that usage in the first month predicts CPAP use in the subsequent two months, an observation recently confirmed by others. ${ }^{17}$

This study also shows that effective CPAP pressure relates to apnoea + hypopnoea frequency and also, independently, to neck size. This latter observation confirms the importance of neck size in the sleep apnoea/hypopnoea syndrome. ${ }^{19}$

1 Young T, Palta M, Dempsey J, Skatrud J, Weber S, Badr S The occurrence of sleep-disordered breathing among The occurrence of sleep-disordered breathing amo
middle-aged adults. $N$ Engl f Med 1993;328:1230-5.

2 Peat JK, Haby M, Spijker J, Berry G, Woolcock AJ Prevalence of asthma in adults in Busselton, Western
Prot Australia. $B M F$ 1992;305:1326-9.

3 Fitzpatrick MF, Martin K, Fossey E, Shapiro CM, Elton RA, Douglas NJ. Snoring, asthma and sleep disturbance in Britain: a community based survey. Eur Respir $\mathcal{F}$ 1993;6:531-5

4 Horn CR, Clark TJH, Cochrane GM. Compliance with inhaled therapy and morbidity from asthma. Respir Med 1990;84:67-70.

5 Dompeling E, Van Grunsven PM, Van Schayck CP, Folgering $\mathrm{H}$, Molema $\mathrm{J}$, Van Weel C. Treatment with inhaled steroids in asthma and chronic bronchitis: longinhaled sterolian in asthma and chronic bronchitis: longterm complia 1992;9:161-6.

6 Mawhinney H, Spector SL, Kinsman RA, Siegel SC Rachelefsky GS, Katz RM, et al. Compliance in clinical trials of two nonbronchodilator, antiasthma medications. Ann Allergy 1991;66:294-9.

7 Whyte KF, Allen MB, Jeffrey AA, Gould GA, Douglas NJ Clinical features of the sleep apnoea/hypopnoea syndrome. $O \mp$ Med 1989;72:659-66.

8 Gould GA, Whyte KF, Rhind GB, Airlie MAA, Catteral JR, Shapiro CM, et al. The sleep hypopnea syndrome. Am Rev Respir Dis 1988;137:895-8.

9 Douglas NJ, Thomas S, Jan MA. The clinical value of polysomnography. Lancet 1992;339:347-50.

10 Carskadon MA, Dement WC, Mitler MM, Roth T, Westbrook PR, Keenan S. Guidelines for the multiple sleep latency test (MSLT): a standard measure of sleepiness. latency test (MSLT):
Sleep 1986;9:519-24.

11 Engleman HM, Cheshire KE, Deary IJ, Douglas NJ Daytime sleepiness, cognitive performance and mood after CPAP therapy for the sleep apnoea/hypopnoea syndrome. Thorax 1993;48:911-4.

12 Thorpy MJ. The clinical use of the multiple sleep latency test. Sleep 1992;15:268-76.

13 Cheshire K, Engleman H, Deary I, Shapiro CM, Douglas NJ. Factors impairing daytime performance in patients with sleep apnea/hypopnea syndrome. Arch Intern Med 1992;152:538-41.

14 Reeves-Hoche MK, Meck R, Zwillich CW. Nasal CPAP. objective evaluation of patient compliance. Am Rev Respir Dis (in press).

15 SPSS-X. User guide. Chicago: McGraw Hill, 1983:604.

16 Cramer JA, Mattson RH, Prevey ML, Scheyer RD, Ouellette UL. How often is medication taken as prescribed? A novel assessment technique. $\mathcal{J} A M A$ 1989;261:3273-7.

17 Kribbs NB, Pack AI, Kline LR, Smith PL, Schwartz AR, Schubert NM, et al. Objective measurement of patterns of nasal CPAP use by patients with obstructive sleep apnea. Am Rev Respir Dis 1993;147:887-95.

18 Krieger J, Kurtz D. Objective measurement of compliance with nasal CPAP treatment for obstructive sleep apnoea with nasal CPAP treatment for obstructi
syndrome. Eur Respir $f 1988 ; 1: 436-8$.

19 Davies RJO, Stradling JR. The relationship between neck circumference, radiographic pharyngeal anatomy, and the obstructive sleep apnoea syndrome. Eur Respir $\mathcal{F}$ 1990;3:509-14. 